ARTÍCULO DE INVESTIGACIÓN

\title{
Eficacia de la Cánula Nasal de Alto Flujo en patologías respiratorias
}

\author{
[Efficiency of High Flow Nasal Cannula in Pediatric Respiratory Pathologies]
}

Dra. Elizabeth Caparó Ingram ${ }^{1}$ (D) , Dr. Rodrigo De Antonio ${ }^{2}$ (D) Dr. José E. Dutari³ ${ }^{3}$ Dr. Manuel Alvarado ${ }^{4}$, Dra. Virginia Díaz $^{3}$, Dr. Marco Donato ${ }^{3}$, Dra. Vanessa Vernaza ${ }^{3}$, Dra. Rosa Flores ${ }^{5}$, Dr. Samuel Roberts ${ }^{5}$, Lic. Osmaldo Muñoz ${ }^{6}$, Licda. Nadia T. Almela Caballero ${ }^{6}$

\begin{abstract}
${ }^{1}$ Neumóloga Pediatra, Hospital del Niño, Dr. José Renán Esquivel, Ciudad Panamá, Panamá. ${ }^{2}$ MD Epidemiólogo, Cand Dr. Salud Pública. ${ }^{3}$ Neumólogo Pediatra ${ }^{4}$ Intensivista Pediatra ${ }^{5}$ Emergenciólogo Pediatra ${ }^{6}$ Terapeuta Respiratorio

Correspondencia:Elizabeth Caparó Ingram / Correo electrónico:elizabethcaparo@hotmail.com
\end{abstract}

Recibido: 4 de febrero 2021

Aceptado: 22 de marzo 2021

Publicado: 24 de junio de 2021.

DOI: $10.37980 /$ im.journal. rspp.20211741

Palabras Clave: cánula nasal de alto flujo, ventilación no invasiva, ventilación mecánica invasiva, insuficiencia respiratoria aguda, unidad de cuidados intensivos pediátricos.

Keywords: high flow nasal cannula, non-invasive ventilation, Invasive mechanical ventilation, acute respiratory failure, pediatric intensive care unit.

Reproducción: Artículo de acceso libre para uso personal e individual. Sujeto a derechos de reproducción para otros usos.

Conflictos de interés: Los autores declaran no tener conflictos de interés. Los autores declaran que han seguido los protocolos de su centro de trabajo sobre la publicación de datos de pacientes. Se obtuvo el consentimiento informado por escrito del paciente para la publicación de este reporte del caso y las imágenes que lo acompañan.

Financiamiento: El autor no declara fuentes externas de financiamiento asociados a este trabajo.

\section{Resumen}

Introducción: En la edad pediátrica hay factores predisponentes importantes que hacen a los niños ser más vulnerables a presentar insuficiencia respiratoria aguda (IRA). Objetivo: Demostrar los beneficios del uso de la cánula nasal de alto flujo (CNAF) en pacientes menores de 5 años con IRA secundaria a enfermedades respiratorias y admitidos a la unidad de cuidados intensivos pediátricos (UCIP). Sujetos y Métodos: Estudio analítico observacional de cohortes mixtas, donde una cohorte está formada por un grupo expuesto a la CNAF evaluada de manera prospectiva, mientras se comparó con una cohorte histórica. Resultados: 138 pacientes fueron admitidos al estudio de los cuales 69 fueron casos manejados con CNAF y 69 fueron controles que no estuvieron expuestos al uso de la CNAF pero sí a ventilación mecánica invasiva. Los días de oxigenoterapia fueron estadísticamente menores en el grupo de casos de 13 (DE 8.01) días a 22 (DE 20.7) días en el grupo control $(P=<0.05)$. Al igual los días de UCIP y los días totales de hospitalización fueron menores en el grupo manejado con CNAF ( $\mathrm{P}=$ 0.011 y $P=0.001$, respectivamente), con una media de tiempo de 10 (DE 7.8) días en UCIP y 17 (DE 9.25) días intrahospitalarios para el grupo de casos versus 14 (DE 10.1) días en UCIP y 28 (DE 23.9) días intrahospitalarios para el grupo control. Conclusión: La CNAF es un método de soporte respiratorio no invasivo, efectivo y fácil de usar en la población pediátrica con IRA secundario a diversas patologías respiratorias.

\section{Abstract}

Introduction: During pediatric ages there are important predisposing factors that make children more vulnerable to present acute respiratory failure (ARF). Objective: Determine the benefits of the use of high flow nasal cannula (HFNC) in patients younger than 5 years with ARF, secondary to respiratory diseases and admitted to the pediatric intensive care unit (PICU). Subjects and Method: Analytical observational study of mixed type cohorts, where a cohort is formed by the group exposed to HFNC and evaluated prospectively, while it was compared with a historical cohort. Results: 138 patients were admitted to the study of which 69 were cases handled with HFNC and 69 were controls who were not exposed to the use of HFNC but to other types of invasive mechanical ventilation. Oxygen therapy days were statistically shorter in the case group from 13 (SD 8.01) days to 22 (SD 20.7) days in the control group $(P=<0.05)$. The length of stay in PICU and the total inpatient days were lower in the group managed with HFNC ( $P=0.011, P=0.001$ ), with a mean time of 10 (SD 7.8) days in PICU and 17 (SD 9.25) inpatient days for the case group versus 14 (SD 10.1) days in PICU and 28 (SD 23.9) inpatient days for the control group. Conclusion: HFNC system is a non-invasive respiratory support method, simple, effective and easy to use in the pediatric population less than 5 years of age with ARF due to various respiratory diseases. 
Introducción

En la edad pediátrica, las particularidades de las vías aéreas y del tórax, así como otros factores físicos, constituyen factores predisponentes importantes que hacen a los niños ser más vulnerables que los adultos a presentar insuficiencia respiratoria aguda (IRA). ${ }^{1}$

El apoyo respiratorio a menudo juega un papel importante en el manejo de niños críticamente enfermos y la ventilación mecánica invasiva (VMI) es la intervención principal en muchos casos. Recientemente, ha habido una tendencia a utilizar la ventilación no invasiva (VNI) en niños críticamente enfermos con una variedad de etiologías de dificultad respiratoria en la unidad de cuidados intensivos pediátricos (UCIP). ${ }^{2}$

La cánula nasal de alto flujo (CNAF) es una modalidad ventilatoria no invasiva de menor complejidad en relación con otros sistemas de soporte respiratorio no invasivo, fue desarrollado inicialmente para recién nacidos prematuros para prevenir atelectasias y apneas como alternativa a la ventilación con presión positiva no invasiva. ${ }^{3}$ Actualmente, esta técnica se está utilizando progresivamente en todos los grupos de edades pediátricas, y cada vez más aceptada por sus efectos positivos sobre la oxigenación, la ventilación y la comodidad del paciente. . $^{411}$

La mayoría de los estudios de la terapia con CNAF han sido realizados en neonatos o en el periodo de postextubación en lactantes con bronquiolitis ${ }^{12}$. Cada vez hay más estudios en población pediátrica que reportan que el uso de la CNAF es un tratamiento eficaz para la insuficiencia respiratoria aguda 4,5,13-15. Sin embargo, los estudios que evalúan la efectividad de la CNAF en niños con diversas etiologías de dificultad respiratoria entre 1 mes y 18 años de edad en UCIP son muy limitados. Una revisión sistemática de Cochrane, del 2014, no pudo encontrar evidencias que permitan determinar la seguridad o efectividad de la terapia con CNAF como una forma de soporte respiratorio en pediatría. ${ }^{4}$

La CNAF como modalidad de apoyo respiratorio no invasivo además de ser más confortable es más fácil de implementar y monitorear, lo que ha permitido su utilización cada vez más expandida en unidades de hospitalización de menor complejidad y unidades de urgencia. Sus principales ventajas son que permite administrar altas concentraciones de oxígeno con una humedad relativa y una temperatura adecuada, mejora la conductancia de las vías respiratorias y la distensibilidad pulmonar y alcanza un cierto nivel de presión positiva continua en las vías respiratorias (CPAP "por sus siglas en inglés"), disminuyendo el trabajo respiratorio. Además, podría reducir el espacio muerto y la resistencia inspiratoria proporcionando un flujo suficiente para igualar o superar el flujo inspiratorio. ${ }^{16-19}$ En el uso clínico para niños, el beneficio más notable de la CNAF es la mejor tolerancia del paciente a la terapia de oxígeno. ${ }^{20} \mathrm{El}$ uso de CNAF probablemente conti- nuará aumentando dado el conocimiento cada vez mayor de esta modalidad de apoyo y facilidad de aplicación. ${ }^{20-22}$

El objetivo de este estudio es demostrar los beneficios del uso de la CNAF en pacientes menores de 5 años con insuficiencia respiratoria aguda, secundario a patologías respiratorias, admitidos en la UCIP.

\section{Pacientes y Método}

El estudio fue realizado con pacientes que tenían diagnóstico de IRA secundario a patologías respiratorias admitidos a la UCIP del Hospital del Niño, Dr. José Renán Esquivel (HNJRE), Panamá, hospital de cuarto nivel de atención, en el período de abril a septiembre 2019, en la unidad de cuidados intensivos 1 y 2; las mismas, son unidades médico-quirúrgicas, de referencia nacional que constan de 14 y 12 camas respectivamente. Los procedimientos realizados en este estudio siguieron los estándares éticos en la Declaración de Helsinki de 1964, revisada en 2008, así como la ley nacional. El estudio fue apoyado por los Proyectos de Investigación Científica de nuestro hospital. El estudio no requirió consentimiento informado ya que no hubo interacción con los pacientes o sus familiares o cuidadores.

Es un estudio observacional analítico de cohortes tipo mixtas o ambidireccional, en donde una cohorte está formada por el grupo de pacientes menores de 5 años ingresados en la UCIP durante el 2019 expuestos a CNAF la cual fue evaluada prospectivamente y se comparó con una cohorte histórica formada por un grupo de pacientes menores de 5 años ingresados en la UCIP durante el periodo enero a diciembre de 2018 que no estuvieron expuestos al uso de CNAF pero si de otras modalidades de ventilación mecánica invasiva, con características similar a los casos, específicamente edad, sexo, diagnóstico de ingreso y valor del índice pediátrico de mortalidad 3 (PIM 3). Para lograr el objetivo se implementó un programa educativo en las diferentes áreas para conocer el mecanismo de acción, las indicaciones, el inicio y el destete, los beneficios, los signos de fracaso, las complicaciones y contraindicaciones del uso de la CNAF. El criterio para iniciar el manejo primario o secundario con la CNAF quedó a cargo del médico tratante del paciente.

Para el cálculo de la muestra se utilizó un nivel de confianza del $95 \%$ y un porcentaje de error máximo aceptado del $5 \%$. La necesidad de ventilación mecánica invasiva con el uso de HFNC fue del 7\% y, por lo tanto, el 93\% restante no lo requirió basado en el estudio de Schibler et al. (23). Según el cálculo de la potencia, se obtuvieron 72 pacientes, de los cuales 36 corresponden a pacientes con uso de HFNC y 36 corresponden a los pacientes con el uso de IMV para mantener la relación 1:1 se requeriría para asegurar el poder del $80 \%$ y $\mathrm{P}<0.05$.

Se incluyeron todos los paciente menores de 5 años, que ingresaron a UCIP del HNJRE, con signos clínicos y/o gaso- 
métricos compatibles con IRA, secundario a patologías respiratorias, manejado con CNAF ya sea de manera primaria (aquellos pacientes que recibieron ventilación con CNAF de manera inicial) o secundaria (aquellos pacientes que recibieron inicialmente soporte ventilatorio invasivo y posterior a la extubación fueron manejados con (NAF) y que tuvieran consignadas todas las variables del estudio en el expediente clínico. Se excluyeron los pacientes menores de 29 días, los pacientes trasladados de otra institución al cuarto de urgencias del HNJRE bajo VNI con CNAF y paciente ya incluido en el estudio.

Durante el estudio, todos los pacientes manejados con CNAF iniciaron con un flujo a $2 \mathrm{l} / \mathrm{kg} / \mathrm{min}$ con su posterior ajuste para mantener una $\mathrm{SO}_{2}$ mayor a $94 \%$. Se consideró fracaso del manejo con CNAF al presentar deterioro clínico en el estado respiratorio o insuficiencia respiratoria que requirió intubación endotraqueal dentro de las primeras 48 horas luego de haber iniciado la CNAF.

Los datos recolectados en ambas cohortes fueron analizados con el programa STATA. Se utilizaron tablas y gráficas para la descripción de las variables de los pacientes del estudio. Para la comprobación de las hipótesis se utilizó parámetros estadísticos según el tipo de variable. En caso de variables cualitativas se utilizó la prueba de $p$ con un nivel de significancia $<0,05$. Para el caso de las variables continuas se utilizó el test de ANOVA y la prueba F. Además se realizó una regresión logística para un análisis bivariado y multivariado.

\section{Resultados}

Las patologías respiratorias comprenden el $40 \%$ de las visitas al cuarto de urgencias pediátricas y el $38 \%$ de los ingresos hospitalarios en la institución, por lo que es importante un tratamiento seguro y efectivo. Durante abril a septiembre 2019 se admitieron 344 pacientes a la sala de cuidados intensivos; 151 (44\%) fueron admitidos a terapia intensiva 1 y $193(56 \%)$ a terapia intensiva 2.

En 2019 se han colocado 271 pacientes en CNAF, de los cuales 96 fueron ingresados al estudio; de estos se excluyeron 27 pacientes por las siguientes razones: 11 continuaban hospitalizados al momento del cierre del estudio, 9 fueron manejados en sala de pediatría general sin ameritar ingreso a terapia intensiva y 7 pacientes tenían los datos incompletos. De esta manera, finalmente se obtuvo un total de 69 casos.

\section{Características generales de los casos y controles}

Se evaluaron los registros según edad, sexo, diagnóstico de ingreso y valor de PIM3. Las características demográficas fueron similares en los casos y controles ( $P=>0.05$ ). El $43.5 \%$ ( $n$ $=30)$ y $44.9 \%(n=31)$ fueron del sexo masculino en el grupo de casos y controles, respectivamente. El $84.0 \%$ de la población correspondió a lactantes menores, con una media de 10 meses (DE 10.1). El $27.5 \%$ de la población tenía comorbili-
Tabla 1. Características de la población

\begin{tabular}{lcccc}
\hline Variable & $\begin{array}{c}\text { Casos } \\
(\mathbf{n}=69)\end{array}$ & $\begin{array}{c}\text { Control VMI } \\
(\mathbf{n}=69)\end{array}$ & $\begin{array}{c}\text { Total } \\
(\mathbf{n}=138)\end{array}$ & Valor P \\
\hline Sexo & $30(44 \%)$ & $31(45 \%)$ & $61(44 \%)$ & $>0.05$ \\
Masculino & & & & \\
Edad & $58(84 \%)$ & $58(84 \%)$ & $116(84 \%)$ & \\
$\quad 1-12$ meses & $6(9 \%)$ & $6(9 \%)$ & $12(9 \%)$ & $>0.05$ \\
$13-24$ meses & $5(7 \%)$ & $5(7 \%)$ & $10(7 \%)$ & \\
$25-60$ meses & & & & \\
& & & & \\
Provincia & $34(49 \%)$ & $34(49 \%)$ & $68(49 \%)$ & $>0.05$ \\
Panamá & $35(50 \%)$ & $35(50 \%)$ & $70(50 \%)$ & \\
Otras & & & &
\end{tabular}

\begin{tabular}{|c|c|c|c|c|}
\hline \multicolumn{5}{|c|}{ Antecedentes personales patológicos } \\
\hline Sí & $20(29 \%)$ & $18(26 \%)$ & $38(28 \%)$ & $>0.05$ \\
\hline \multicolumn{5}{|l|}{ Diagnóstico } \\
\hline Neumonía & $59(86 \%)$ & $59(86 \%)$ & 118 & \multirow{3}{*}{$>0.05$} \\
\hline Bronquiolitis grave & $7(10 \%)$ & $7(10 \%)$ & $14(10 \%)$ & \\
\hline Crisis asmática grave & $2(3 \%)$ & $2(3 \%)$ & $2(3 \%)$ & \\
\hline \multicolumn{5}{|l|}{ PIM 3} \\
\hline Muy alto & $1(1 \%)$ & $0(0.0 \%)$ & $1(1 \%)$ & \multirow{4}{*}{$>0.05$} \\
\hline Alto & $12(17 \%)$ & $13(19 \%)$ & $25(18 \%)$ & \\
\hline Moderado & $30(44 \%)$ & $30(44 \%)$ & $60(44 \%)$ & \\
\hline Bajo & $26(38 \%)$ & $26(38 \%)$ & $52(38 \%)$ & \\
\hline
\end{tabular}

Caso y Controles (razón de 1:1), fueron pareados según edad, sexo, diagnóstico de ingreso y valor de PIM3 (Muy alto: > 30\%, Alto: 15-29\%, Moderado: 5-14.9\%, Bajo: 1-4.9\%, Muy Bajo <1\%).

dades asociadas; entre las más frecuentes fueron cardiopatía congénita, asma bronquial y prematuridad. El diagnóstico más común en general fue neumonía ( $n=59,86 \%$ ), luego bronquiolitis grave $(n=7,10 \%)$ y en menor proporción crisis asmática grave $(n=2,3 \%)$ (Tabla 1).

La CNAF se utilizó como soporte respiratorio primario en el $63 \%$ de los pacientes; de estos, el $29 \%$ inició su manejo en UCIP, el $19 \%$ en urgencias y el $15 \%$ en salas de pediatría general. El 37\% restante fue colocado de manera secundaria posterior a la extubación. Los días promedios del uso del alto flujo fueron 3 días (DE 2). La terapia fue exitosa en 59 (86\%) pacientes, mientras que $10(14 \%)$ pacientes necesitaron escalar a ventilación mecánica, en su mayoría en las primeras 24 horas. Las causas de fracaso incluyeron apnea, aumento de trabajo respiratorio, estridor inspiratorio, desaturación y bradicardia (Tabla 2). En el grupo que requirió intubación endotraqueal, 3 pacientes iniciaron su manejo en UCIP, 3 pacientes iniciaron luego de la extubación, 2 pacientes en sala de medicina general y 2 pacientes en el cuarto de urgencias pediátricas. En el presente estudio, no encontramos variables estadísticamente significativas para correlacionar con el fracaso de la terapia.

En los pacientes manejados con CNAF, la frecuencia respiratoria tuvo su mayor descenso a los 60-120 minutos de haber iniciado su manejo con un promedio de 51 respiraciones por minuto (rpm) a $42 \mathrm{rpm}$. Igualmente la frecuencia cardíaca 
Tabla2. Características asociadas al inicio, fracaso y complicaciones del uso de la Cánula nasal de alto flujo

\begin{tabular}{ll}
\hline & $\begin{array}{c}\text { CNAF } \\
(\mathrm{n}=69)\end{array}$ \\
\hline Inicio de la CNAF & \\
Postextubación & $26(37 \%)$ \\
Sala de medicina & $10(15 \%)$ \\
UCIP & $20(29 \%)$ \\
Urgencias & $13(19 \%)$
\end{tabular}

\begin{tabular}{ll} 
Fracaso de CNAF & \\
\hline Sí & $10(15 \%)$
\end{tabular}

\begin{tabular}{lc} 
Causa fracaso CNAF & \\
\hline Apnea & $1(1 \%)$ \\
Aumento de trabajo respiratorio & $7(10 \%)$ \\
Desaturación y bradicardia & $1(1 \%)$ \\
Estridor inspiratorio & $1(1 \%)$
\end{tabular}
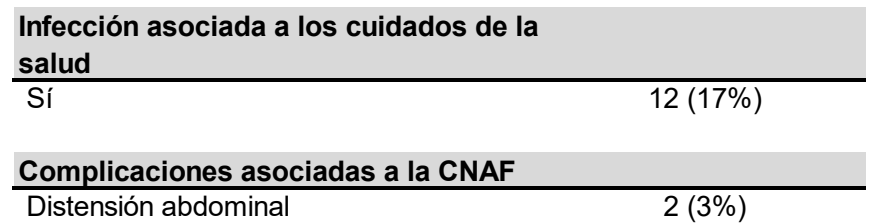

\begin{tabular}{ll} 
Muerte \\
\hline Sí & $4(6 \%)$ \\
\hline
\end{tabular}

CNAF: cánula nasal de alto flujo, UCIP: unidad de cuidados intensivos pediátricos, VMI: ventilación mecánica invasiva.

presentó mayor descenso a los 60-120 minutos de 147 latidos por minuto (lpm) a 135 lpm.

La CNAF fue bien tolerada en todos los pacientes. De los 69 pacientes estudiados, solo 2 presentaron complicaciones menores (distensión abdominal) atribuidas al uso de la CNAF. Ningún paciente presentó traumatismo en la mucosa nasal, epistaxis, neumotórax, neumomediastino ni enfisema subcutáneo. Un total de 3 pacientes (4.3\%) fallecieron en el grupo sin respuesta a la CNAF, mientras que 1 (1.44\%) falleció en el grupo de respuesta. Ninguno de los fallecimientos ocurrió durante su manejo con CNAF; estos pacientes fallecieron en promedio después de 8 días de haber escalado a VMI.

\section{Análisis comparativo de ambas cohortes}

El promedio del $\mathrm{pH}$ y $\mathrm{PaCO}_{2}$ previo al soporte respiratorio fue 7.4 (7.15-7.54) y 49 (25-82) en el grupo de casos y 7.28 (6.97.55) y 55 (20-128) en el grupo de control, respectivamente $(P=<0.05)$. A pesar que en la determinación de la saturación de oxígeno se evidenció aumento de la misma en ambos grupos, este incremento no fue estadísticamente significativa para los pacientes manejados con CNAF.

Los días de oxigenoterapia fueron estadísticamente menores en el grupo de casos de 13 (DE 8.01) días a 22 (DE 20.7) días en el grupo control $(P=<0.05)$. Al igual los días de UCIP y los días totales de hospitalización fueron menores en el grupo manejado con CNAF ( $\mathrm{P}=0.011$ y $\mathrm{P}=0.001$, respectiva-
Figura 1. Días totales de oxígeno (A) Días en UCIP y total de días hospitalizados en CNAF y los controles (B). CNAF: Cánula nasal de alto flujo *Estadísticamente significativo $(P=<0.05)$.

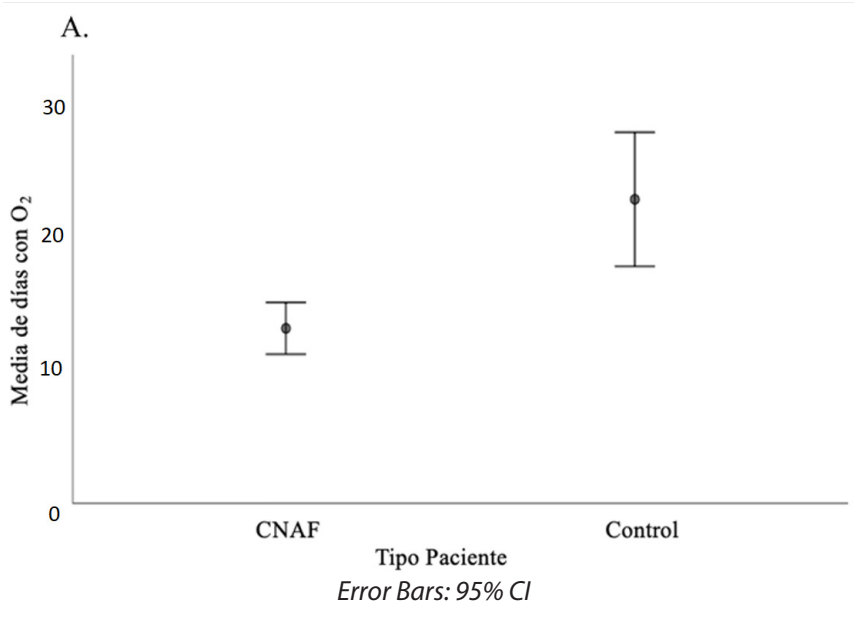

B.

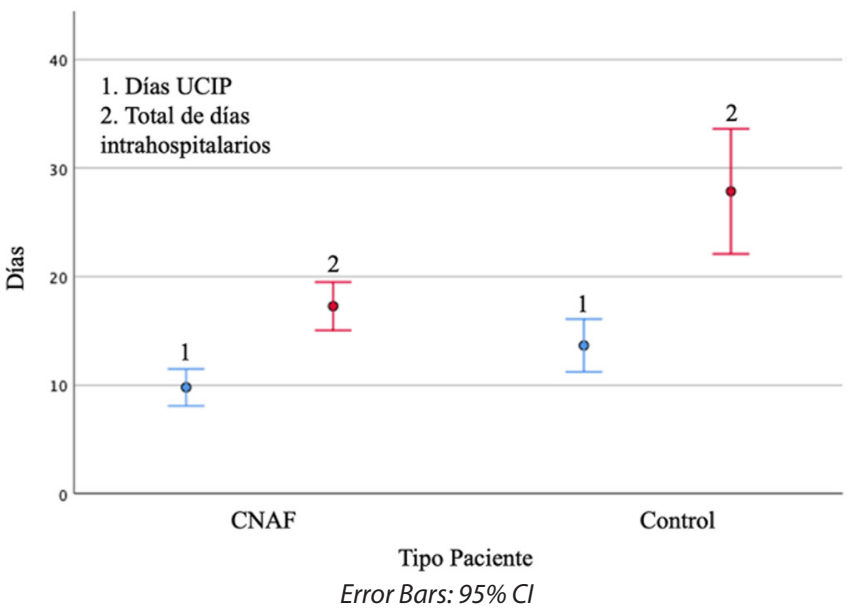

mente), con una media de tiempo de 10 (DE 7.8) días en UCIP y 17 (DE 9.25) días intrahospitalarios para el grupo de casos versus 14 (DE 10.1) días en UCIP y 28 (DE 23.9) días intrahospitalarios para el grupo control (Fig. 1).

Las complicaciones, en general, asociadas a la VMI también predominaron en el grupo control con un $31.9 \%$ versus un $10.1 \%$ en el grupo de casos. No hubo diferencias significativas en la tasa de mortalidad entre los dos grupos.

\section{Discusión}

La importancia del estudio radica en que hasta la fecha en nuestro país no existe ningún estudio similar. Realizar este estudio nos ayudó a evaluar los beneficios más significativos asociados al uso de la CNAF en nuestra unidad. A pesar que existieron limitaciones, se decidió realizar un estudio de cohorte mixta observacional analítico, donde se confirmo que la CNAF es una terapia eficaz que mejora los signos de dificultad 
respiratoria en las primeras horas de tratamiento; además, es una terapia segura ya que no se observaron efectos adversos mayores que pusieran en peligro la vida del paciente.

Durante el estudio, todos los pacientes manejados con CNAF iniciaron con un flujo a $2 \mathrm{l} / \mathrm{kg} / \mathrm{min}$ con su posterior ajuste para mantener una $\mathrm{SO}_{2}$ mayor a $94 \%$. La tasa de éxito en pacientes menores de 5 años con insuficiencia respiratoria secundaria a patologías respiratorias en nuestro estudio fue de $86 \%$, similar a lo descrito en la literatura por Kamit et al. ${ }^{2}$, Oto et al. ${ }^{14} \mathrm{y}$ Wing et al. ${ }^{24}$ siendo $87 \%, 80 \%$ y $84 \%$, respectivamente.

Mayfield et al. ${ }^{25}$ reportó que la frecuencia cardíaca descendió significativamente dentro de $60 \mathrm{~min}$ de $158 \mathrm{lpm}$ a 144 Ipm al igual que la frecuencia respiratoria cayó de 54 rpm a 51 rpm. Igualmente, Oto et al. ${ }^{14}$ demostró una caída en la frecuencia cardíaca de $137 \mathrm{lpm}$ a $128 \mathrm{lpm}$ a los 30 minutos. Asimismo, la frecuencia respiratoria se redujo de 41 a $38 \mathrm{rpm}$ a los 30 minutos. En nuestro estudio, al igual que en otros estudios adicionales $2,17,26$ la frecuencia cardiaca y la frecuencia respiratoria disminuyeron luego de iniciar la terapia, teniendo su mayor descenso a los 60-120 minutos.

Al evaluar el fracaso de la CNAF, definido como la necesidad de intubar al paciente dentro de las primeras 48 horas luego de haber iniciado su manejo, de los 10 (14.5\%) pacientes que fallaron más de la mitad lo hizo dentro de las primeras 24 horas. El porcentaje de pacientes que no tuvieron éxito se asemeja a estudios publicados previamente 2,14,26,27. Guillot et al. ${ }^{28}$ realizó un estudio prospectivo observacional en una UCIP sugiriendo la CNAF como tratamiento de primera línea en IRA y encontrando como único factor asociado al fracaso de la terapia una $\mathrm{PaCO}_{2}$ elevada $(\mathrm{P}=0.046)$. Kelly et al. ${ }^{27}$ en un estudio retrospectivo de 498 niños que acudieron a dos cuartos de urgencias pediátricos con dificultad respiratoria encontraron que además de la $\mathrm{PaCO}_{2}$ elevada ( $>50 \mathrm{~mm}$ $\mathrm{Hg}$ ), el $\mathrm{pH}$ disminuido $(<7.3)$ y la frecuencia respiratoria a su llegada mayor del percentil 90 para la edad se asociaron de forma independiente con la necesidad de intubación dentro de las 24 horas de iniciada la terapia con CNAF. A pesar de realizar un análisis bivariado y multivariado en busca de factores asociados al fracaso de la CNAF, ningún resultado fue estadísticamente significativo, lo cual puede ser debido a un número de muestra pequeño.

Dos pacientes presentaron distensión abdominal asociada al uso de la CNAF; ninguno cursó con complicaciones mayores ni infecciones. Hasta la fecha estudios previos reportan una baja tasa de complicaciones asociadas al alto flujo 2,14,17,26. Tres estudios informaron distensión abdominal en niños con CNAF, lo que indica que se debe tener cuidado en pacientes con patología intraabdominal ${ }^{8}$. El resultado principal del estudio fue evaluar los días de estancia en terapia intensiva, obteniendo resultados estadísticamente significativos a favor del manejo con CNAF, teniendo una media de 10 (DE 7.8) días en el grupo de casos versus 14 (DE 10.1) días en el grupo control. Al igual, se pudo observar una disminución de los días intrahospitalarios en total entre el grupo de casos y el grupo control. Estos resultados son diferentes a lo expuesto por Ballestero et al. ${ }^{17}$. Wing et al. ${ }^{24}$ y Mayfield et al. ${ }^{25}$. La diferencia muy probablemente sea debida a que ninguno de los tres estudios previos comparó la CNAF con VMI. Un estudio retrospectivo realizado en Turquía en el 2016 por Can et al. 12 encontró que el uso de la CNAF como soporte respiratorio primario disminuyó los días en UCIP y los días totales de hospitalización en pacientes que no requirieron intubación o VMI.

El manejo con ventilador mecánico tiene un costo elevado, al igual que el tratamiento de las complicaciones asociadas a la intubación endotraqueal y la ventilación mecánica invasiva. Por el contrario, la CNAF ofrece ventajas clínicas y eventos adversos mínimos que permiten reducir la necesidad de asistencia respiratoria invasiva; y por ende disminuir los costos hospitalarios.

El estudio tiene un número de limitaciones. Primero, es un estudio observacional de cohorte de tipo mixto con control histórico donde los sesgos son inevitables. Además, es un estudio de un solo centro donde nuestros hallazgos pueden no aplicarse a otras instituciones y que tiene un número de muestra relativamente pequeño.

\section{Conclusión}

Podemos concluir que el sistema de CNAF es un método de soporte respiratorio no invasivo simple, efectivo y fácil de usar en la población pediátrica menor de 5 años con insuficiencia respiratoria aguda debido a diversas enfermedades respiratorias. Pudimos determinar que es una modalidad segura, evaluando su respuesta con indicadores clínicos como frecuencia cardíaca, frecuencia respiratoria y saturación de oxígeno. Los pacientes que respondieron al tratamiento mostraron una mejoría en el tratamiento dentro de las primeras dos horas de uso. Sin embargo, la mejora inicial puede no indicar el éxito final debido al hecho de que el monitoreo continuo es importante para la predicción temprana de la falla y, por lo tanto, evitar retrasar el uso de otro sistema de soporte de ventilación. Además, es importante mencionar la baja frecuencia de eventos adversos asociados con el uso de CNAF.

La CNAF representa una alternativa como soporte respiratorio no invasivo con beneficios no solo para el paciente sino también económicamente para la institución con respecto al manejo con ventilación mecánica invasiva. Los resultados de este estudio nos permiten considerar otro tipo de terapia respiratoria no invasiva y, por lo tanto, disminuir la duración de la estadía en la UCIP con una mejor estrategia rentable en pacientes seleccionados adecuadamente. Se debe considerar realizar un estudio tipo ensayo clínico aleatorizado en el futuro para corroborar la eficacia y seguridad del uso de CNAF. 


\section{Agradecimiento}

Agradecimiento a todo el equipo del servicio de terapia respiratoria y al Lic. Julio Nieto del laboratorio.

\section{Referencias}

1. Hammer J, Eber E. The Peculiarities of Infant Respiratory Physiology. En: Hammer J, Eber E. Pediatric Pulmonary Function Testing. Switzerland: Karger; 2005.p 2-7.

2. Kamit F, Berna A, Anil M et al. Predictive factors for the outcome of high flow nasal cannula therapy in a pediatric intensive care unit: Is the SpO2/FiO2 ratio useful? J Crit Care. 2018; 44:436-444. doi: 10.1016/j.jcrc.2017.09.003. Epub 2017 Sep 6.

3. Demoule A, Rello J. High flow oxygen cannula: the other side of the moon. Intensive Care Med. 2015;41(9):1673-1675. doi: 10.1007/s00134-015-3855-5. Epub 2015 Jun 3.

4. Mayfield S, Jauncey-Cooke J, Hough J, Schibler A, Gibbons K, Bogossian F. High-flow nasal cannula therapy for respiratory support in children. Cochrane Database Syst Rev. 2014;3:1-14. doi: 10.1002/14651858.CD009850.pub2

5. Wegner A, Cespedes $\mathrm{P}$, Loreto $\mathrm{M}$ et al. Cánula nasal de alto flujo en lactantes: experiencia en una unidad de paciente crítico. Rev Chil Pediatr. 2015;86(3):173-181. doi: 10.1016/j.rchipe.2015.06.003. Epub $2015 \mathrm{Jul} 6$.

6. Montecchia F, Midulla F, Papoff P. A flow-leak correction algorithm for pneumotachographic work-of-breathing measurement during high-flow nasal cannula oxygen therapy. Med Eng Phys. 2018;54:32-43. doi: 10.1016/j.medengphy.2018.02.004. Epub 2018 Mar 2.

7. Schmid F, Manfred D, Ballmann M. The use of high-flow nasal cannula (HFNC) as respiratory support in neonatal and pediatric intensive care units in Germany - A nationwide Survey. Respir Med. 2017;131:210-214. doi: 10.1016/j.rmed.2017.08.027. Epub 2017 Sep 1

8. Mikalsen I, Davis P, Øymar K. High flow nasal cannula in children: a literature review. Emerg Med Clin. 2016;24:93. doi: 10.1186/ s13049-016-0278-4.

9. Lin S, Liu K, Lin Z, Lin P. Does high-flow nasal cannula oxygen improve outcome in acute hypoxemic respiratory failure? A systematic review and meta-analysis. Respir Med. 2017;131:58-64. doi: 10.1016/j.rmed.2017.08.005. Epub 2017 Aug 9.

10. Kline J, Kalburgi S, Halley T. High Flow Nasal Cannula Therapy for Bronchiolitis Across the Emergency Department and Acute Care Floor. Emerg Med Clin. 2018;19(1):40-44. doi.org/10.1016/j. cpem.2018.02.001

11. Wegner A. High flow nasal cannula in children. Neumol Pediatr. 2017; 12(1):5-8.

12. Can F, Berna A, Anil M et al. Impact of High-flow Nasal Cannula Therapy in Quality Improvement and Clinical Outcomes in a Non-invasive Ventilation Device-free Pediatric Intensive Care Unit. Indian J Pediatr. 2017;54:835-841. doi: 10.1007/s13312017-1145-8. Epub 2017 Jul 11

13. Pilar F, López Y. Oxigenoterapia de alto flujo. An Pediatr Contin. 2014;12(1):25-29.

14. Oto A, Erdoğan S, Boşnak M. Oxygen therapy via high flow nasal cannula in pediatric intensive care unit. Turk J Pediatr.
2016;58(4):377-382. doi: 10.24953/turkjped.2016.04.005.

15. Mayfield S, Jauncey J, Bogossian F. A case series of paediatric high flow nasal cannula therapy. Aust Crit Care. 2013;26 (4):189192. doi: 10.1016/j.aucc.2013.05.004.

16. López J, Pilar J. Modalidades de ventilación no invasiva en niños. En: Medina A, Pons M, Martinón F. Ventilación no invasiva en pediatría. Segunda edición. España: Ergon; 2009. p 49-60.

17. Ballestero Y, De Pedro J, Portillo N, Martinez O, Arana E, Benito J. Pilot Clinical Trial of High-Flow Oxygen Therapy in Children with Asthma in the Emergency Service. J Pediatr. 2018;194:204-210 e3. doi: 10.1016/j.jpeds.2017.10.075. Epub 2018 Jan 11

18. Alexander J. Advanced respiratory support on the paediatric Ward. J Paediatr Child Health. 2017;27(5):238-241. doi.org/10.1016/j.paed.2017.01.007

19. Haq I, Gopalakaje S, Fenton A, McKean M, O'Brien C, Brodlie $M$. The evidence for high flow nasal cannula devices in infants. Paediatr Respir Rev. 2014;15(2):124-134. doi: 10.1016/j. prrv.2013.12.002. Epub 2013 Dec 17.

20. Morley S. Non-invasive ventilation in paediatric critical care. Paediatr Respir Rev. 2016;20:24-31. doi: 10.1016/j.prrv.2016.03.001

21. Lee J, Rehder K, Williford L, Cheifetz I, Turner D. Use of high flow nasal cannula in critically ill infants, children, and adults: a critical review of the literatura. Intensive Care Med. 2013;39(2):247-257. doi: 10.1007/s00134-012-2743-5. Epub 2012 Nov 10.

22. Slain KN, Shein SL, Rotta AT. The use of high-flow nasal cannula in the pediatric emergency department. J Pediatr Rio J. 2017;93(Suppl1):36-45. doi: 10.1016/j.jped.2017.06.006. Epub 2017 Aug 15.

23. Schibler A, Pham TMT, Dunster KR et al. Reduced intubation rates for infants after introduction of high-flow nasal prong oxygen delivery. Intensive Care Med. 2011;37(5):847-52. doi: 10.1007/ s00134-011-2177-5.

24. Wing R, James C, Maranda LS, Armsby CC. Use of High-Flow Nasal Cannula Support in the Emergency Department Reduces the Need for Intubation in Pediatric Acute Respiratory Insufficiency. Pediatr Emer Care. 2012;28 (11):1117-23. doi: 10.1097/ PEC.0b013e31827122a9

25. Mayfield S, Bogossian F, O'Malley L, Schibler A. High-flow nasal cannula oxygen therapy for infants with bronchiolitis: Pilot study. J Paediatr Child Health. 2014;50(5):373-8. doi: 10.1111/ jpc.12509.

26. Monteverde E, Fernández A, Ferrero F et al. Oxigenoterapia con cánula nasal de alto flujo en lactantes con infección respiratoria baja aguda. Experiencia en hospitales de la Ciudad de Buenos Aires. Arch Argent Pediatr. 2019;117(5):286-293.

27. Kelly GS, Simon HK, Sturm JJ. High-Flow Nasal Cannula Use in Children With Respiratory Distress in the Emergency Department. Pediatr Emer Care. 2013;29(8):888-92. doi: 10.1097/PEC. 0b013e31829e7f2f.

28. Guillot $\mathrm{C}$, Le Reun $\mathrm{C}$, Behal $\mathrm{H}$ et al. First-line treatment using high-flow nasal cannula for children with severe bronchiolitis: Applicability and risk factors for failure. Arch Pediatr. 2018;25(3)213-18. doi: 10.1016/j.arcped.2018.01.003. Epub 2018 Mar 15. 